\section{P06.10 LACTIC ACID DAMPENS INFLAMMATORY RESPONSES ELICITED BY MICROBIAL TLR AGONISTS FROM VAGINAL AND CERVICAL EPITHELIAL CELLS}

1,2,3,4 G Tachedjian* ${ }^{1,2} \mathrm{AC}$ Hearps, ${ }^{1,3} \mathrm{D}$ Srbinovski, ${ }^{1} \mathrm{D}$ Tyssen, ${ }^{1,3} \mathrm{M}$ Aldunate, ${ }^{1} \mathrm{R}$ Gugasyan, ${ }^{5} \mathrm{DJ}$ Anderson, ${ }^{6}$ RA Cone. ${ }^{1}$ Centre for Biomedical Research, Burnet Institute; ${ }^{2}$ Department of Infectious Diseases, Monash University; ${ }^{3}$ Department of Microbiology, Monash University; ${ }^{4}$ Department of Microbiology \& Immunology at the Peter Doherty Institute for Infection and Immunity, The University of Melbourne; ${ }^{5}$ Department of Obstetrics and Gynecology, Boston University School of Medicine; ${ }^{6}$ Department of Biophysics, Johns Hopkins University

\subsection{6/sextrans-2015-052270.311}

Introduction Vaginal lactobacilli are associated with favourable sexual health outcomes and acidify the vagina to $\mathrm{pH}<4.0$ by producing $0.3-1 \% \mathrm{D}$ and $\mathrm{L}$ isomers of lactic acid (LA). Epithelial cells that line the vagina and cervix have barrier and immune functions in the lower female reproductive tract (FRT). Here we investigate the immune modulatory effects of L-LA on lower FRT epithelial cells that might influence HIV susceptibility.

Methods The effect of apically applied L-LA $(0.3 \%$ w/w, pH3.9) was assessed on vaginal (VK2), endocervical (End), ectocervical (Ect) epithelial cell lines and primary ectocervical cells grown in transwells. Elicited immune mediators were quantified following apical stimulation with toll-like receptor (TLR) agonists \pm L-LA by flow cytometry and luminex-based assays.

Results L-LA had little impact on FRT epithelial cell viability. Stimulation of FRT epithelial cell lines with the TLR3-agonist poly (I:C) (PIC) induced high-levels of pro-inflammatory cytokines (IL-6/IL-8), and their variable induction with TLR agonists Pam (3) CSK(4) (TLR1/2) and lipopolysaccharide (TLR4). Conversely, L-LA treatment significantly reduced PIC-induced IL-6 ( 30 -fold) and IL-8 (3-4.5-fold, $\mathrm{p} £ 0.03)$ secretion, compared to PIC-only treated FRT epithelial cell lines. Irrespective of TLR stimulation, L-LA elicited a $4-11$-fold $(p<0.01)$ increase in the anti-inflammatory cytokine IL-1RA in FRT epithelial cell lines. Neither $0.3 \%$ L-LA at neutral $\mathrm{pH}$ nor acidity alone $(\mathrm{HCl}, \mathrm{pH}$ 3.9) elicited the abovementioned effects indicating that immune modulation is mediated by the protonated form of L-LA and is not due to low $\mathrm{pH}$. L-LA also reduced PIC-induced secretion of RANTES and MIP3a in all cells, associated with recruitment of HIV target cells to the mucosa. Similar anti-inflammatory effects of L-LA were observed in primary ectocervical cells.

Conclusion L-LA found in lactobacillus-dominated vaginal microbiota elicits an anti-inflammatory effect on lower FRT epithelial cells and dampens inflammation induced by microbial TLR agonists suggesting a role in mitigating inflammationinduced HIV susceptibility at the vaginal mucosa.

Disclosure of interest statement This study was funded by NHMRC Project APP1088564. No pharmaceutical grants were received in the development of this study.

\section{P06.11 THE IMPACT OF PERIODIC PRESUMPTIVE TREATMENT FOR VAGINAL INFECTIONS ON THE VAGINAL MICROBIOME AMONG WOMEN PARTICIPATING IN THE PREVENTING VAGINAL INFECTIONS TRIAL}

${ }^{1,2} \mathrm{JE}$ Balkus* ${ }^{1} \mathrm{C}$ Andac, ${ }^{1,2 \mathrm{DN}}$ Fredricks, 30 Anzala, 3J Kimani, 4J Schwebke, 15 Srinivasan, 5J Lee, 2,3RS McClelland. ${ }^{1}$ Fred Hutchinson Cancer Research Center; ${ }^{2}$ University of Washington; ${ }^{3}$ University of Nairobi; ${ }^{4}$ University of Alabama at Birmingham; ${ }^{5}$ University of Arkansas for Medical Sciences

10.1136/sextrans-2015-052270.312
Background A randomised trial of monthly periodic presumptive treatment (PPT) with intravaginal metronidazole $750 \mathrm{mg}$ plus miconazole $200 \mathrm{mg}$ reduced bacterial vaginosis (BV) by $35 \%$ compared to placebo. We further assessed the effect of the intervention on detection of select bacterial species in the vaginal microbiome.

Methods HIV-uninfected, non-pregnant women aged 18-45 years from the US and Kenya were randomised to receive PPT or matching placebo for 5 consecutive nights each month for 12 months. Vaginal fluid specimens were collected every other month using Dacron swabs and tested using species-specific quantitative PCR assays that target the 16S rRNA gene. Relative risks $[R R]$ were generated using generalised estimating equations with a log link and exchangeable correlation structure to separately assess the effect of the intervention on species detection.

Results Of 234 women enrolled, 221 (94\%) had specimens for analysis (PPT $\mathrm{n}=110$; placebo $\mathrm{n}=111$ ). The proportion of follow-up visits with individual species detected was lower in the PPT arm versus placebo for: BVAB1 (13.8\% vs. 23.7\%; $\mathrm{RR}=0.60,95 \%$ CI $0.39-0.93)$, BVAB2 (30.7\% vs. 42.5\%; RR $=0.72 ; 95 \%$ CI $0.55-0.95)$, BVAB3 $(22.9 \%$ vs. $31.0 \% ; \mathrm{RR}=$ $0.75,95 \%$ CI $0.54-1.03)$, Atopobium vaginae $(59.7 \%$ vs. 72.7\%; RR $=0.82,95 \%$ CI 0.71-0.94), Leptotrichia/Sneathia (49.4\% vs. $60.6 \%$; RR $=0.81,95 \%$ CI $0.68-0.97)$, and Megasphaera species $(26.8 \%$ vs. $43.8 \%$; RR $=0.61,95 \%$ CI $0.46-$ 0.82 ). Lactobactillus crispatus and L. jensenii were more frequently detected in the PPT arm (L. crispatus: $31.8 \%$ vs. $26.7 \%$, $\mathrm{p}=0.19$; L. jensenii: $31.8 \%$ vs. $25.1 \% ; \mathrm{p}=0.07)$. However, these increases were not statistically significant. The prevalence of Gardnerella vaginalis and L. iners during follow-up was high (90\% and $91 \%$, respectively) and did not differ by arm.

Conclusions Use of monthly PPT for one year significantly reduced $\mathrm{BV}$ prevalence as well as colonisation with a number of bacterial species strongly associated with BV. The role of PPT to improve vaginal health should be considered.

Disclosure of interest statement R. S. M. has received honoraria for invited lectures and consulting as well as donated study product for this trial from Embil Pharmaceutical Company. R. S. M. currently receives research funding from Hologic/Gen-Probe. J. E. B. received honoraria from Symbiomix, Inc for consulting and donated reagents from Hologic/Gen-Probe. J. S. has received consultancy payments from Akesis, Hologic, Symbiomix, and Starpharma, and has grants/pending grants from Akesis, BD Diagnostic, Hologic, Cepheid, Quidel, Symbiomix, Starpharma, and Viamet. All other authors declare that they do not have a commercial or other association that might pose a conflict of interest.

\section{P06.12 HUMAN IL-36 GAMMA AS AN INDICATOR OF VAGINAL INFECTION AND PROMOTER OF MUCOSAL INFLAMMATION}

M Herbst-Kralovetz* ${ }^{*} \mathrm{~S}$ Winkle, A Throop. University of Arizona, College of MedicinePhoenix

\subsection{6/sextrans-2015-052270.313}

Introduction IL-36 $\gamma$ (also designated as IL-1F9) has been recently identified and belongs to the IL-1 family of cytokines. Despite expression of IL-36 $\gamma$ at other mucosal sites, it has not previously been reported in the vaginal or cervical epithelium. Overall, there is a paucity of information regarding the induc-

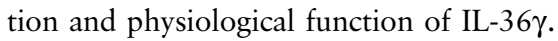

